\title{
Application of spiking neural networks for modelling the process of high-temperature hydrogen production in systems with gas-cooled reactors
}

\author{
Sergey O. Starkov ${ }^{1}$, Yury N. Lavrenkov ${ }^{2}$ \\ 1 Obninsk Institute for Nuclear Power Engineering, National Research Nuclear University "MEPhI", 1, Studgorodok, Obninsk, Kaluga reg., \\ 249040 Russian Federation \\ 2 Kaluga Branch of the Bauman Moscow State Technical University, 2, Bazhenova str., Kaluga, Kaluga reg., 248000 Russian Federation
}

Corresponding author: Yury N. Lavrenkov (georglawr@yandex.ru)

Academic editor: Yury Kazansky • Received 10 March 2019 • Accepted 23 May 2019 • Published 21 June 2019

Citation: Starkov SO, Lavrenkov YN (2019) Application of spiking neural networks for modelling the process of high-temperature hydrogen production in systems with gas-cooled reactors. Nuclear Energy and Technology 5(2): 129-137. https://doi.org/10.3897/ nucet.5.36474

\begin{abstract}
Hydrogen energy is able to solve the problem of the dependence of modern industries on fossil fuels and significantly reduce the amount of harmful emissions. One of the ways to produce hydrogen is high-temperature water-steam electrolysis. Increasing the temperature of the steam involved in electrolysis makes the process more efficient. The key problem is the use of a reliable heat energy source capable of reaching high temperatures. High-temperature gas-cooled reactors with a gaseous coolant and a graphite moderator provide a solution to the problem of heating the electrolyte. Part of the heat energy is used for producing electrical energy required for electrolysis. Modern electrolyzers built as arrays of tubular or planar electrolytic cells with a nuclear energy source make it possible to produce hydrogen by decomposing water molecules, and the working temperature control leads to a decrease in the Nernst potential. The operation of such facilities is complicated by the need to determine the optimal parameters of the electrolysis cell, the steam flow rate, and the operating current density. To reduce the costs associated with the process optimization, it is proposed to use a low-temperature electrolysis system controlled by a spiking neural network. The results confirm the effectiveness of intelligent technologies that implement adaptive control of hybrid modeling processes in order to organize the most feasible hydrogen production in a specific process, the parameters of which can be modified depending on the specific use of the reactor thermal energy. In addition, the results of the study confirm the feasibility of using a combined functional structure made on the basis of spiking neurons to correct the parameters of the developed electrolytic system. The proposed simulation strategy can significantly reduce the consumption of computational resources in comparison with models based only on neural network prediction methods.
\end{abstract}

\section{Keywords}

Spiking neural networks, high-temperature gas-cooled reactors, electro-optical neural commuting system, hydrogen production forecasting, centralized global parallel search circuit

* Russian text published: Izvestiya vuzov. Yadernaya Energetika (ISSN 0204-3327), 2019, n. 1, pp. 143-153. 


\section{Introduction}

Like electricity, hydrogen is a high-quality energy carrier that can be produced using a variety of materials that determine the process of obtaining, distributing, storing and transporting this type of fuel. One of the purest methods for producing hydrogen is the dissociation of water molecules, but low-temperature electrolysis requires a greater amount of electrical energy and is an expensive process (Gupta 2008). The article describes the process of neural network management of a system of connected electrolytic cells for modeling high-temperature water steam electrolysis. Generation of steam of the required temperature and electrolysis occur in such conditions due to the energy produced by high-temperature gas-cooled reactors (Cacuci 2010). As a result, hydrogen is produced both with the use of thermal energy and its partial conversion into electricity. The development of new systems requires a testing process in order to determine the amount of hydrogen produced and estimate the designed installation efficiency at given geometrical parameters (tubular or planar electrolytic cell (EC)), numbers of cells in electrolysis assemblies (channels or chambers, depending on the EC shape), parameters of porous electrodes, electrolysis temperature, current density and voltage. Physical testing is complicated by the need to create high temperatures and the use of materials and alloys that are resistant to high temperatures and changes in mechanical properties due to the formation of hydrides and methane because of the absorption of hydrogen by the installation materials.

Below is a description of the electrolysis unit designed to obtain the necessary physical parameters for simulations and the developed neural network architecture. The development of a neural network configuration algorithm is discussed in accordance with a specific feature of the simulation system, i.e., the availability of a source of information about the simulated process.

\section{Electrolytic cell development}

To eliminate the need for a physical experiment at power generation plants, it is proposed to use a system of coupled electrolyzers (Tomilova 1984), the layout of which is shown in Fig. 1.

The amount of hydrogen produced is controlled by changing the following parameters:

1. Limiting the current through each cell by means of a current mirror with a reflection coefficient of $1: 8$, made on the basis of a matched pair of TIP36C bipolar pnp-transistors with SQP emitter resistors $(0.1$

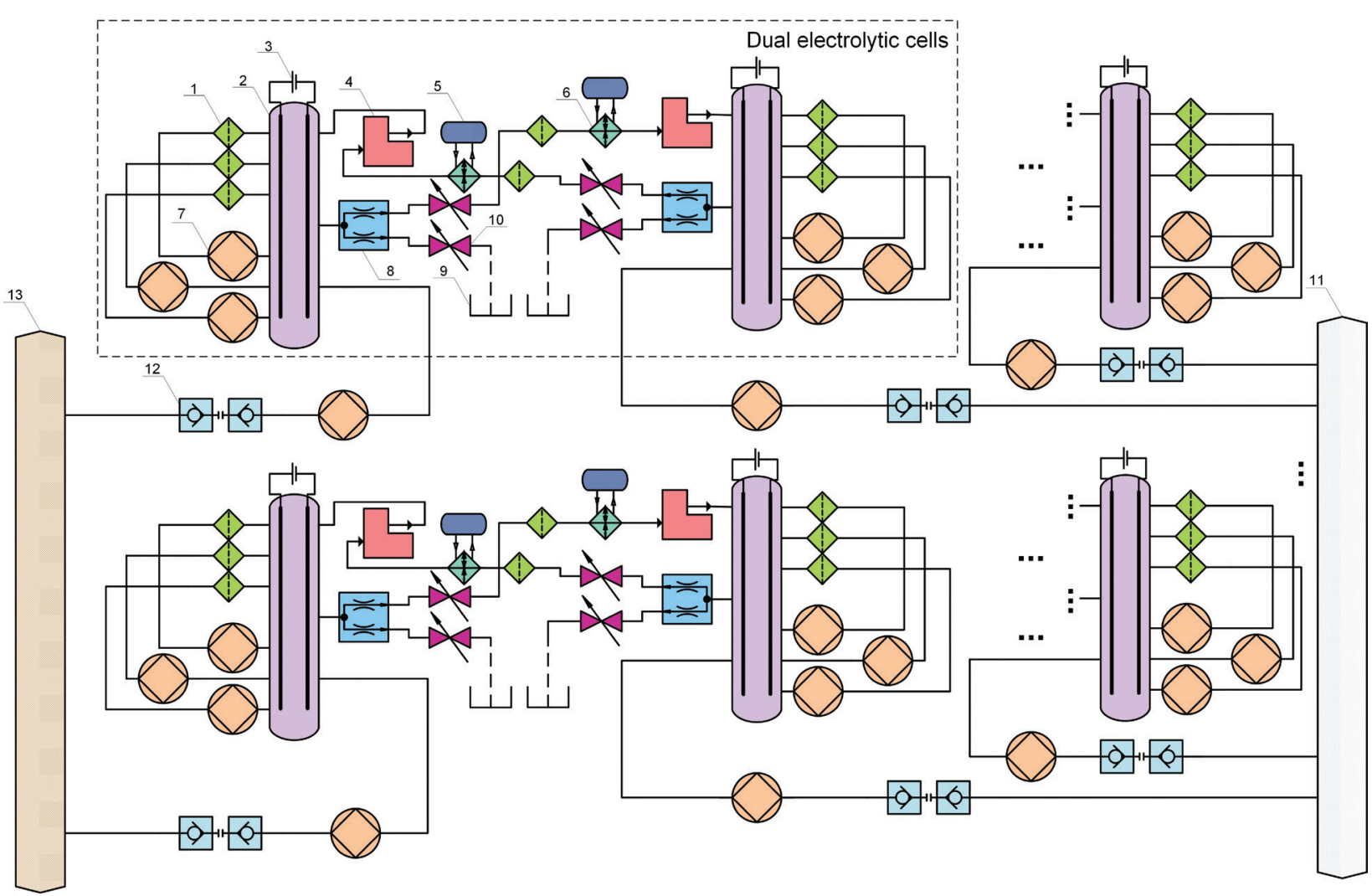

Figure 1. Structural diagram of the installation for predicting the amount of hydrogen produced: 1 . Filter to remove particles of electrodes; 2. Electrolytic cell with graphite electrodes; 3. Electrolyzer power supply system; 4. Peristaltic dosing pump; 5. Liquid resistor; 6. Electrolyte heat exchanger; 7. Diaphragm pump; 8. Flow divider; 9. Electrolyte drain tank; 10. Solenoid valve; 11. Tank with $\mathrm{CuSO}_{4}$ solution; 12 . Connector; 13. Distilled water tank 
Ohm). As a control current source, a Wilson current mirror based on TIP3055 npn-transistors (Gray Paul et al. 2009) was used (a total of two parameters per dual electrolytic cell (DEC)).

2. Regulating the pulsed electrolysis parameters by changing the pauses between direct current pulses, determining the moments of application of reverse current pulses, and superimposing an alternating current (AC) on a direct current (DC) according to a circuit with an additional inert electrode (the AC source is an ET190E electronic transformer). This process is necessary to control the morphology of electrolytic deposits on the electrodes, the redistribution of crystallization centers and the dissolution of microdendrites and knobs, which contributes to grinding hydrogen bubbles and facilitates their separation from the cathode. Control is carried out by switching the electrolytic cell into the H-bridge made on 2SC3281 (npn) and 2SA1302 (pnp) transistors. (A total of four parameters per DEC: one parameter for each $\mathrm{H}$-bridge and one parameter to determine time intervals for the AC imposition).

3. Changing the electrolyte temperature in a cell by heating the liquid resistors using CCFL inverters, made on the OZ9910GN pulse-width converter chip, and a group of FDS8958A dual field-effect transistors. To perform local electrolyte heating, an internal network of KNP-200 wire resistors (resistant to pulsations) with a nominal value of $100 \mathrm{Ohm}$ is used: the resistors are evenly distributed throughout the cell volume and are heated in pulsed mode by discharging a battery of electrolytic capacitors (six $4700 \mu \mathrm{F}$ capacitors with a rated operating voltage of $100 \mathrm{~V}$ ) through a BT138-600 thyristor. (A total of four parameters per DEC: two for controlling the inverters, two for controlling the heaters.)

4. Regulating the electrolyte concentration in the cells by peristaltic pumps (with a maximum flow rate of $40 \mathrm{ml} / \mathrm{min}$ ) and diaphragm pumps (with a maximum flow rate of $21 / \mathrm{min}$ ). The electrolyte is diluted with distilled water from reservoir 13 (see Fig. 1) or increased in concentration by adding copper sulfate solution from reservoir 11 (see Fig. 1) and subsequently pumped among the cells. The flow rate is regulated by pulse-width modulators made on the basis of an NE555 timer, Schottky rectifier (STPS41H100CT) and $n$-channel MOSFET transistor (STP80NF70) (Crecraft and Gergely 2002) (a total of six parameters per DEC: four for controlling the pumps, two for controlling the electromagnetic valves).

5. Bubbling control using three diaphragm pumps (a total of six variables per DEC).

Therefore, to control electrolysis in one dual electrolytic cell, it is necessary to control 22 parameters that completely determine the modeling process. The required number of dual cells is determined by the parameters of coordination with a real nuclear facility for hydrogen production (Yan and Hino 2011).
The system input parameters, which are fed to the neural network input, are data on the steam electrolysis system configuration and the reactor thermal energy distribution balance (OECD 2006). By controlling the electrolysis process parameters using a spiking neural network, it is possible to simulate the process of high-temperature steam electrolysis at a given scale, which determines the proportionality of hydrogen production for this model as compared to high-temperature nuclear plants for combined electric energy/hydrogen generation. Neural network control methods make it possible to synthesize the optimal current form and pulsed electrolysis parameters to ensure the minimum time required to achieve a permissible prediction error.

\section{Designing the neural network system for developing a hybrid modeling strategy}

To control the parameters of the considered electrolytic cell system, it is proposed to use a spiking neural network (Gerstner and Kistler 2002). Signals among neurons are propagated as short electrical pulses, i.e., spikes. The force of stimuli has a direct impact on the number of spikes and the intervals between them. The basis of the spiking neural network construction is a neuron (Fig. 2 ), the architecture of which is based on the principles of reverberant circuit operation (Maass and Bishop 2001). The neuron is made of two composite computing modules: a modular lattice computing structure, which allows an increase in computing elements (Cichocki and Amari 2002), and a cascade neural network structure (Haykin 1999). The spiking network has dynamic properties that make it possible to organize an information processing algorithm not only by setting weights but also by changing the connections between neurons. A population of neural elements is able to adaptively adjust the oscillation process that synchronizes the neural network elements, due to which it is possible to generate spikes at certain time intervals. As a result, the neuron activity is determined by the input signals, the activity at the previous stage of operation as well as the state of the other neurons included in the reverberation circuit.

An artificially implemented axonal transport mechanism plays an important role in maintaining a stable spike generation process (Sterratt et al. 2011) (Fig. 3). By controlling the characteristics of channels for transferring information among neurons, it is possible to turn the network to asynchronous operation mode without a significant change in the weighting coefficients, followed by additional training. This architectural feature minimizes the likelihood of creating separate neuron clusters within the spiking network with its local synchronization system.

The basis of the switching system is a magnetic amplifier with an AC output (Gottlieb 1998), designed from two modified transformers (B78386-P1116-A), in 


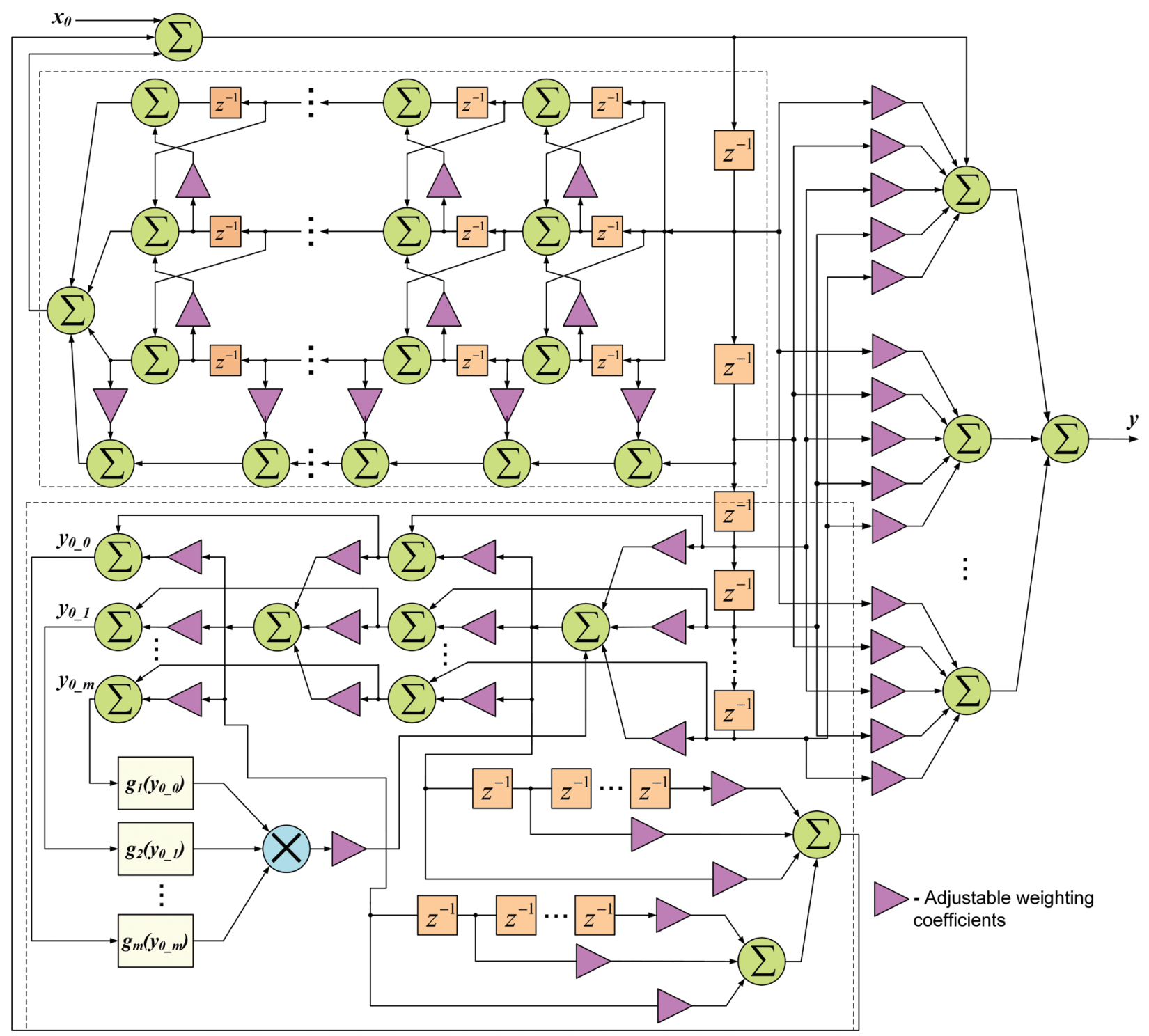

Figure 2. Structural diagram of the neural element for constructing a spiking network

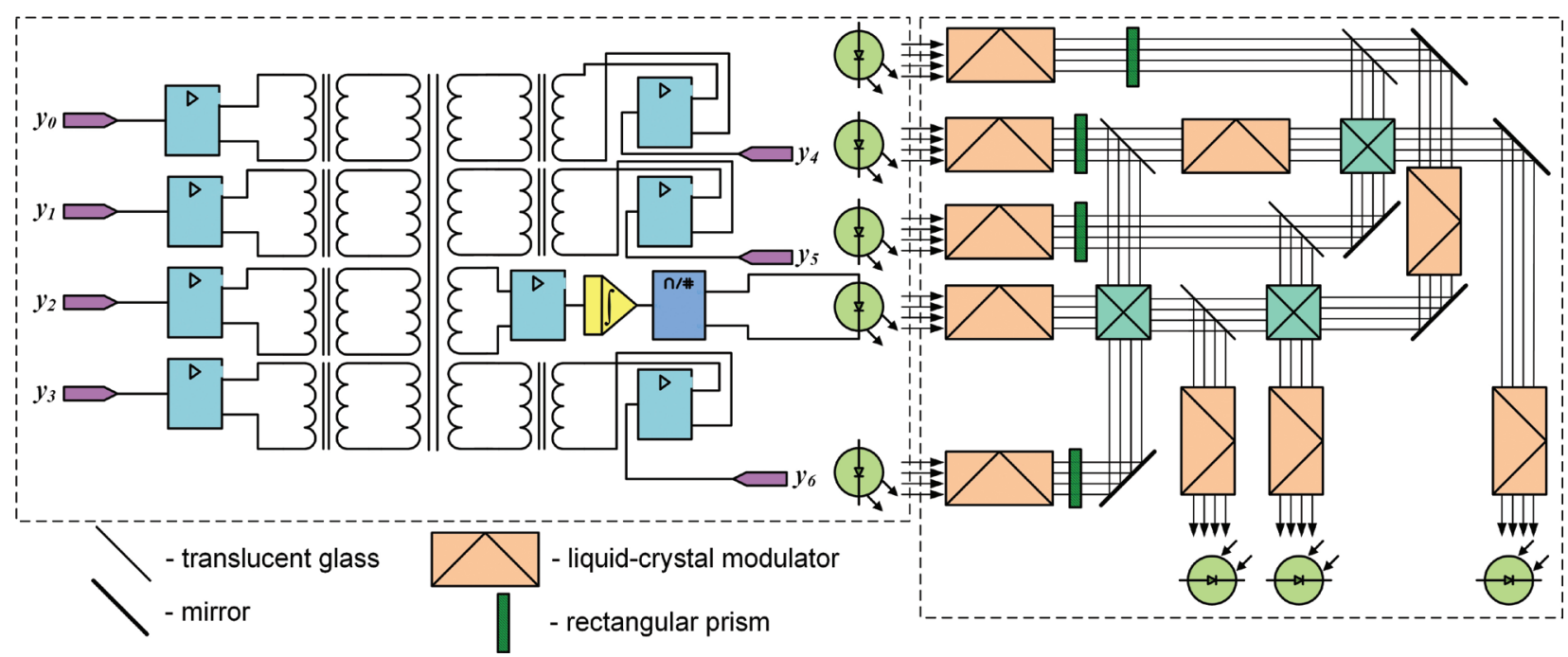

Figure 3. Structural diagram of the electro-optical transducer 


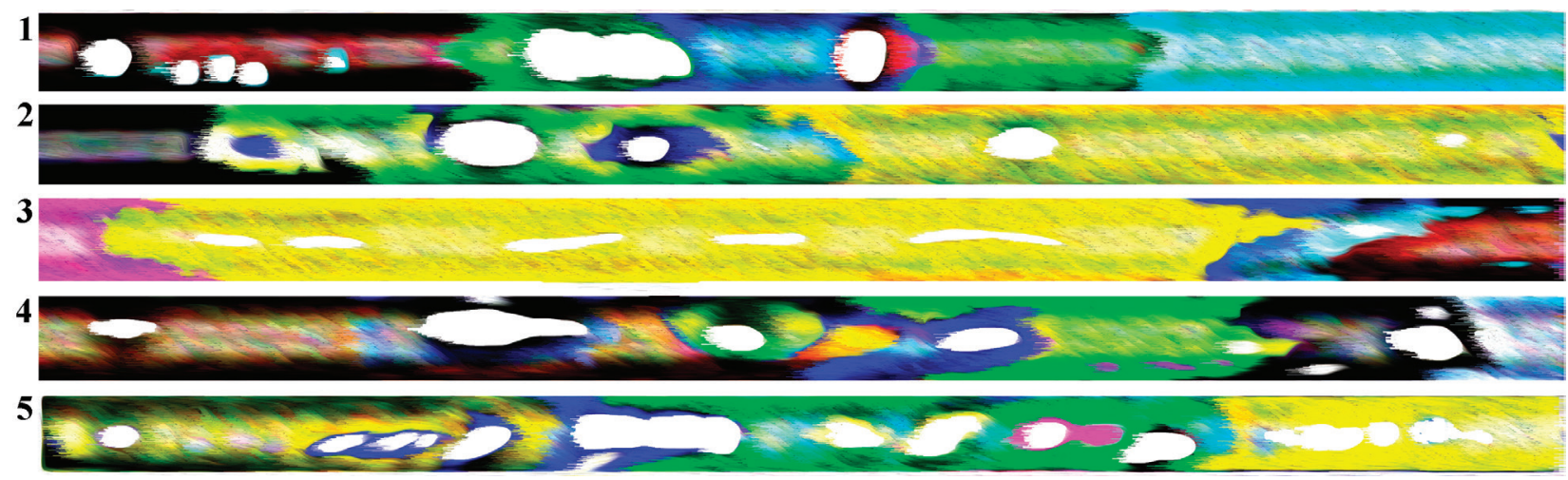

Figure 4. Optical signals after passing an array of prismatic elements

which the magnetic circuit is replaced by a ferrite rod core (M2000NM). Signals were fed from a population of seven neurons through a TDA8932T amplifier and an isolating EI14 audio transformer to a magnetic amplifier to form an output signal (Crecraft and Gergely 2002). To expand the possibilities of signal processing, an optoelectronic system is used, which determines the strategy of interaction among spike populations. The output signal is converted by means of an analog measuring circuit made on the cascade switching of an LM3914 chip, an integrating circuit based on the LM358 operational amplifier and a laser diode into light pulses, the parameters of which are determined by the sequence of spike pulses. The optical signals are processed by the system from an array of liquid crystal screens, cubic X-prisms and rectangular dichroic prisms (Saleh and Teich 2007). The basic cell of the optical system (see Fig. 3, the righthand subsystem) consists of a set of optical modulators, prisms and mirrors. The parameters of the liquid crystal optical filter (degree of darkening, sensitivity, delay be-

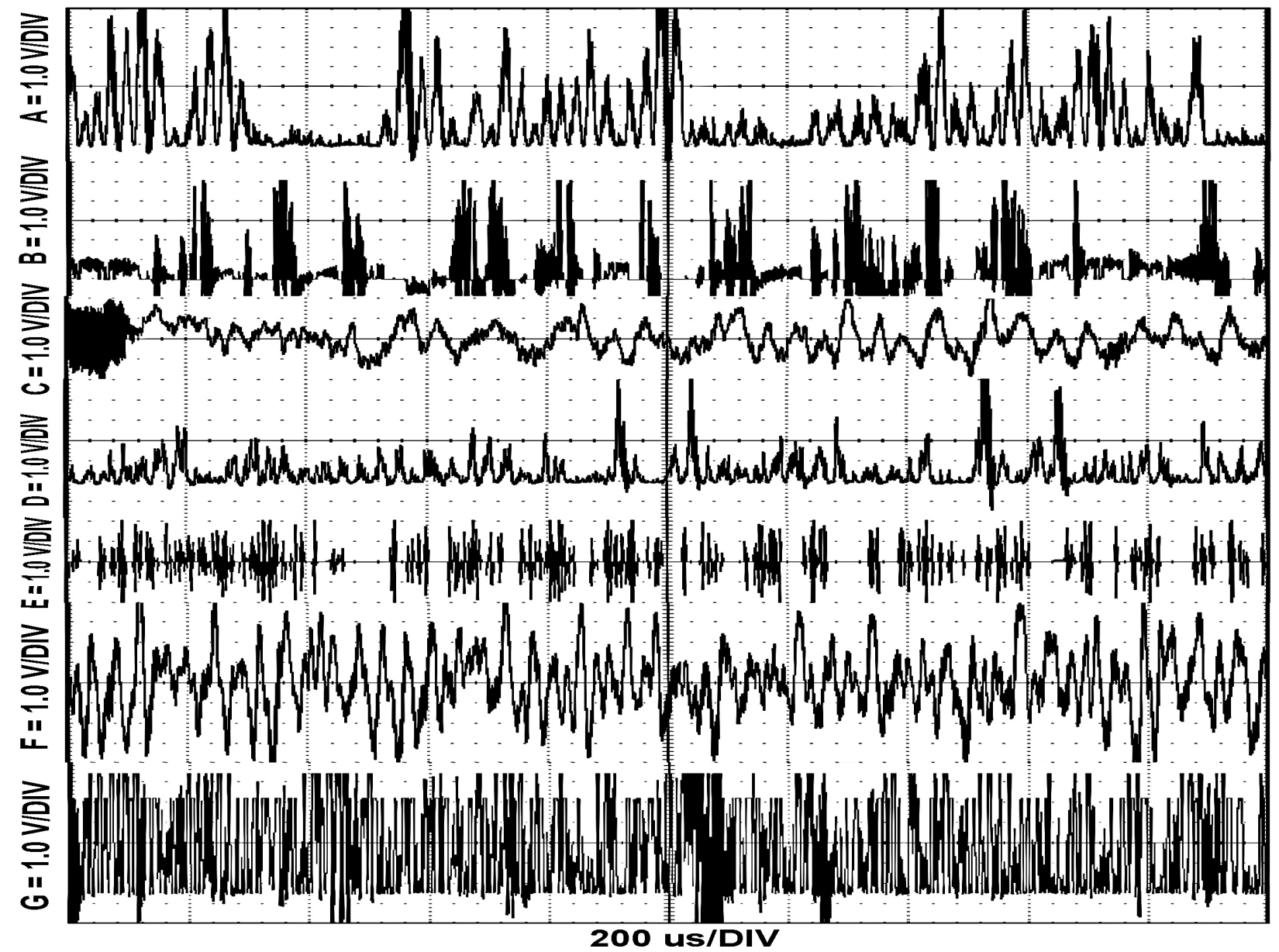

Figure 5. Neural group input signals 


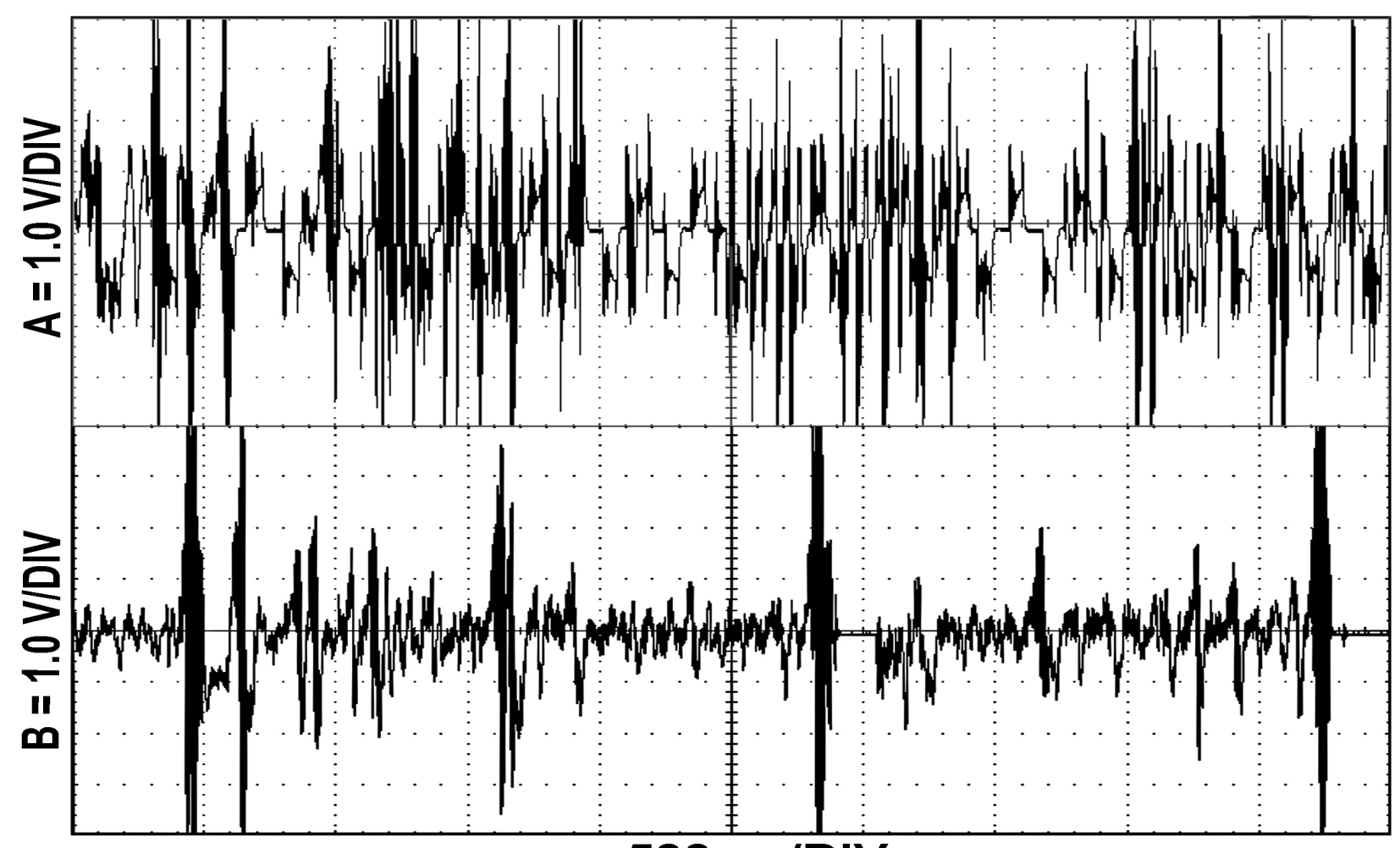

500 us/DIV

Figure 6. Neural group output signals

fore changing the crystal orientation) are related to the adjustable parameters of the spike network. After passing through the optical array, the signal is subjected to optoelectronic conversion by means of the OPT101 photodiode array. The photodiode output signal is an actuating signal for the voltage-controlled generator, which is made on the ICL8038 chip with a TL082A operational amplifier. After passing through the integrating chain, the spike spreads through the network. Figure 4 shows a photograph of samples of light signals from a single optical cell. Samples 1 and 2 exemplify neuron interactions through the optical medium to the training point when an initial network rhythm is observed. Samples 3 and 4 show pulses of different intensity (passing spikes of different amplitudes) as well as combinations of pulses into groups (the light spot area expansion), indicating that the neural network attempts to stimulate photodetectors located in a certain neighborhood of the photodetector line, which makes it possible, due to sequential stimulation, to obtain a spike with the required characteristics. Sample 5 shows the interaction of spiking neurons after training.

The considered spiking neuron network design is scalable, which makes it possible to design a network with the required computing power by simply combining optical and electronic modules. The constructive elements are designed in such a way that a population of seven neurons together with a single cell of an electro-optical converter can form spike pulses with a given information coding system (Moss and Gielen 2001). This property of individual neurons is necessary to maintain the functioning of the entire network when using optical infor- mation processing and switching systems that can distort the transferred pulses. Let us consider the functioning of the neural network structure. Input signals are generated using functional periodic dependencies based on nested functions (Wai-Kai Chen 2005), which reflect the initial parameters of the problem.

Figure 5 shows possible input signals for the spike network; each signal is fed to the input of the corresponding neural element. After the signals are combined by the electro-optical system, the output signal has the form shown in Fig. 6. Signal A in the figure corresponds to the output signal for the network that did not pass the training procedure, in which the weights and parameters of the electro-optical system were initialized with random numbers (a vector generalized lagged Fibonacci generator was used). Signal B shows the pulses produced after setting and configuring variable parameters.

The output signal of the neural network is represented by means of a modified pulse phase modulation method (Maass and Bishop 2001). The system involves signals from four neural elements of the spike network (Fig. 7). To unambiguously determine the pulse, it is necessary that its amplitude be at least $2 \mathrm{~V}$ with a length of less than 190 $\mu \mathrm{s}$. The moment of time, which determines the beginning of the information sequence decoding, is counted when three spikes appear in Neuron C and Neuron D is not active (mark 0, Fig. 7). The activity of Neuron D is synchronizing for the whole group of four signals; the appearance of a spike in Channel D is combined with the activity of Neuron C. If the spikes in $\mathrm{C}$ and $\mathrm{D}$ are not matched by more than $150 \mu \mathrm{s}$, the information is extracted from Chan- 


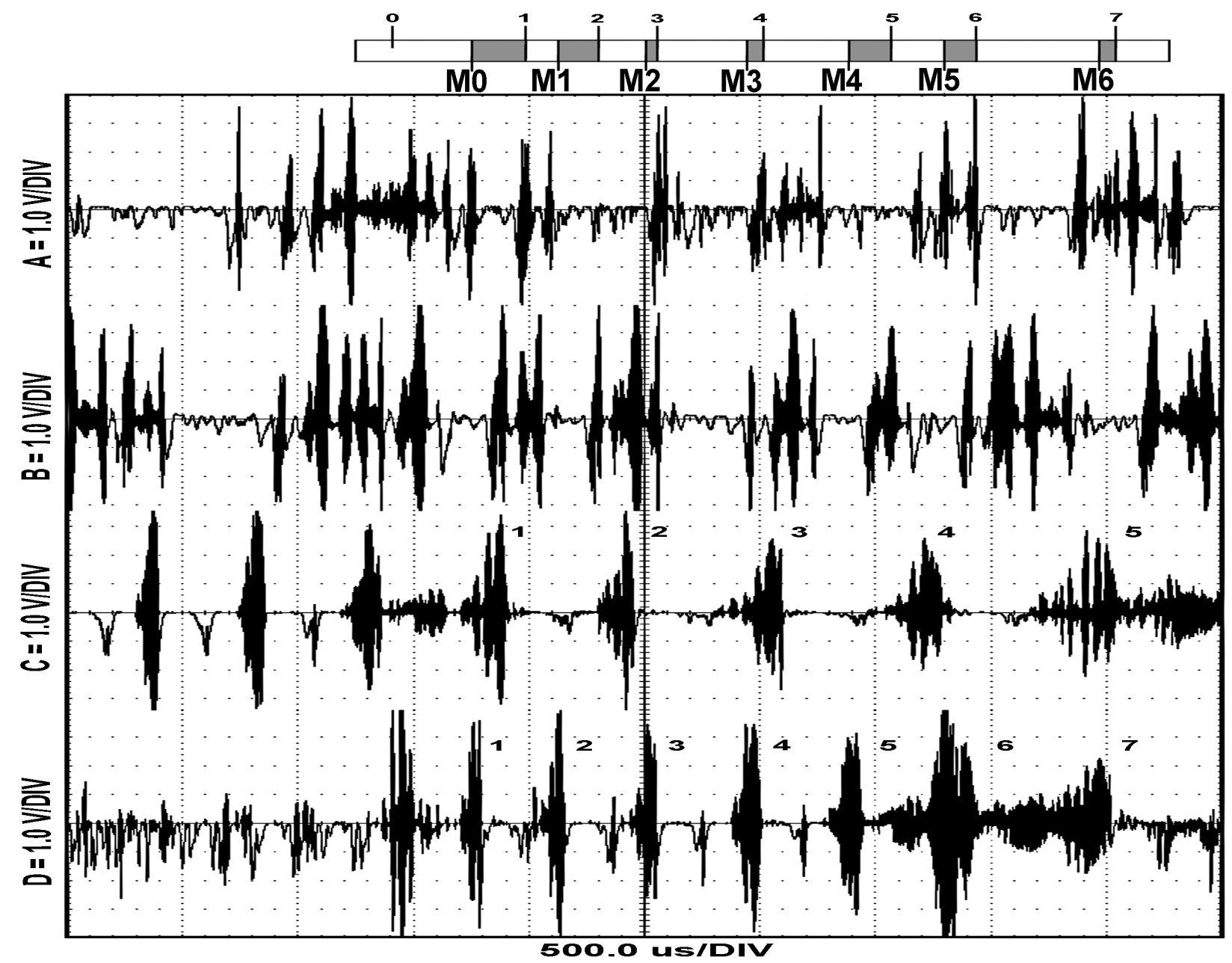

Figure 7. Coding information in a sequence of pulses from four neurons
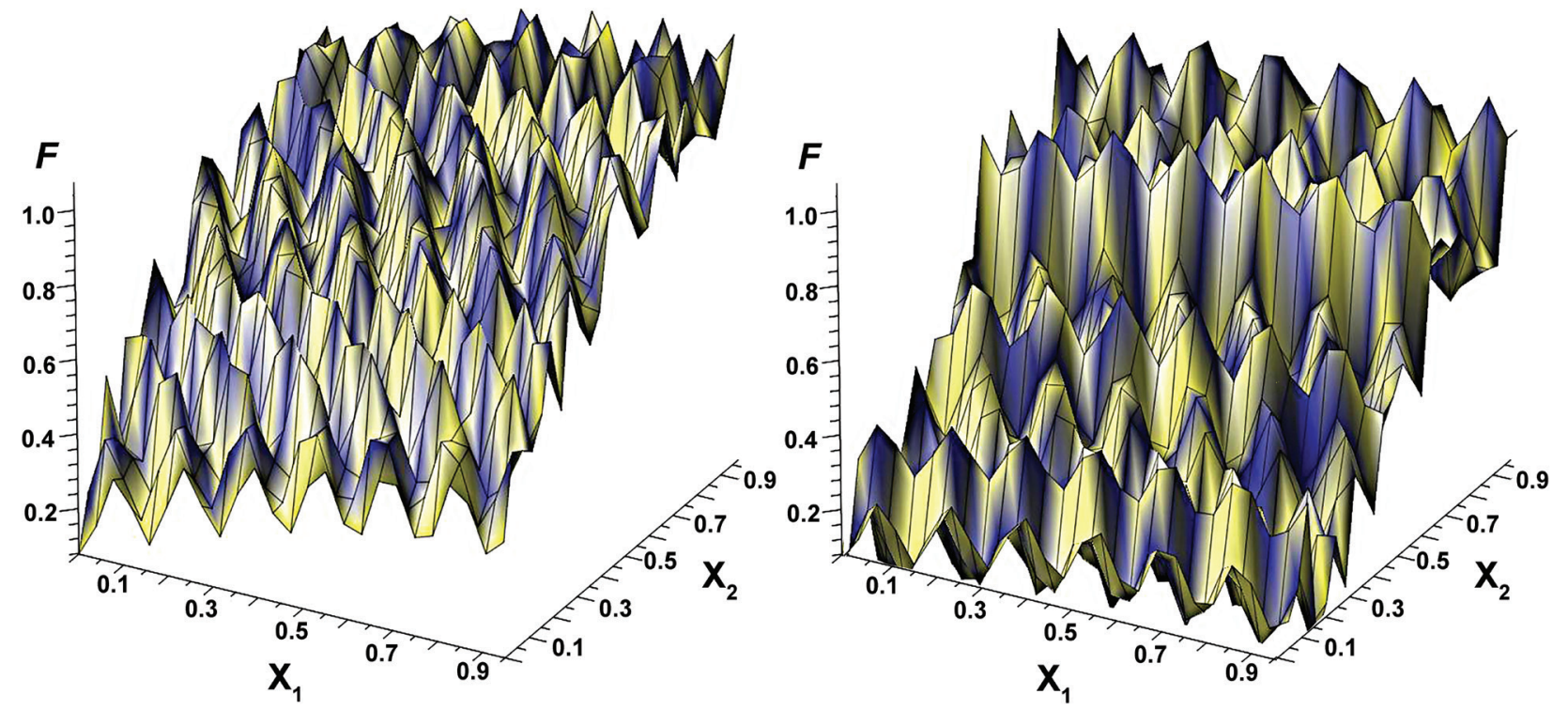

Figure 8. Hydrogen yield dependences on system parameters

nel A, otherwise from Channel B. Information extraction is based on the time stamping of a spike pulse occurring in the observed neural activity channel (A or B).
In Figure 7, information extraction starts from Mark 0. Spikes D. 1 and C. 1 are mismatched by less than 150 $\mu \mathrm{s}$; therefore, information is extracted from Channel A 
(mark the pulse time in Channel A (Mark 1, Fig. 8) after the synchronizing spike in Channel D (Mark M0, Fig. 7)). Further, Spikes D.2 and C.2 are mismatched by more than $150 \mu \mathrm{s}$; therefore, information is extracted from Channel B (mark the pulse time in Channel B (Mark 2, Fig. 7) after the synchronizing spike in Channel D (Mark M1, Fig. 7)). The procedure is repeated until the next moment of the beginning of reading information or termination of activity in Synchronizing Channel D.

The variable time interval between the synchronizing spike and the pulse of the corresponding neuron is used to represent the output neural network signal.

\section{Configuring the spike neural network system and evaluating the forecast results}

The considered structural elements of the spike neural network include variable parameters: weighting factors in the neuron, parameters of the lattice and cascade structures and parameters of the electro-optical system. The initial network topology is a structure consisting of three layers of spike neurons. A centralized global parallel search scheme was used as the training algorithm (Strongin et al. 2013). Each neural population was implemented on an ATxmega128A1 microcontroller. In this scheme, these populations can be considered as "testing processors (TP)" (Strongin et al. 2013). The "control processor (CP)" transfers test points (parameters of each neural population) to the TPs. After testing, all information is collected in the CP, which concludes about the effectiveness of potential parameters for the entire spike network. As an algorithm for generating test points, a scattered search algorithm was used (Greshilov 2014).

The efficiency of hybrid hydrogen production modeling using a high-temperature electrolysis system was estimated based on plotting the amount of gas produced depending on the gas-cooled reactor characteristics and the electrolyzer parameters (Fig. 8). The parameters $x_{1}$ (a set of electrolytic cell parameters) and $x_{2}$ (reactor parameters) are defined as the sum of the weighted parameters characteristic of a particular hydrogen production method:

$$
x_{i, i=1,2}(k)=\sum_{j=1}^{Q} w_{j} \Phi_{j}(r, \sigma), \quad r=\|k-c\|, \Phi_{j}(r, \sigma)=\left(\frac{r}{\sigma}\right)^{2} \log \left(\frac{r}{\sigma}\right),
$$

where $w_{j}$ are weighting coefficients; $Q$ is the number of parameters; $r$ is the distance between the input vector $k$ and the eigenvector $c$; $\sigma$ is the scale parameter. The weighted parameters adapt the system for a wide range of nuclear systems. All variables are normalized in accordance with the maximum physical limitations. The applicate shows the amount of hydrogen produced. A comparison of the modeling process (Fig. 8b) with the results obtained in practical installations (Yan and Hino 2011, OECD 2006) (Fig. 8a) allows us to conclude that neural network systems can be used to control the hybrid modeling processes.

\section{Conclusion}

The development of new electrolytic cell configurations to increase the efficiency of nuclear energy is an important issue when hydrogen is used as the basis for clean energy. Difficulties arising from the design of hydrogen cogeneration systems in nuclear power engineering can be successfully overcome with the use of neural network decision-making methods, which make it possible to quickly assess the effectiveness of structural changes in the system. The results obtained during the work confirm the effectiveness of intelligent technologies that implement adaptive control of hybrid modeling processes in order to organize the most feasible production of hydrogen for a specific process, the parameters of which can be modified depending on the specific use of the reactor thermal energy. In addition, the results of the study confirm the feasibility of using a combined functional structure made on the basis of spiking neurons to correct the parameters of the proposed electrolytic system. The proposed simulation strategy can significantly reduce the consumption of computational resources in comparison with models based only on neural network prediction methods.

\section{References}

- Cacuci DG (2010) Handbook of Nuclear Engineering. Springer, 3574 pp. https://doi.org/10.1007/978-0-387-98149-9

- Cichocki A, Amari SI (2002) Adaptive Blind Signal and Image Processing: Learning Algorithms and Applications. Wiley, 586 pp. https://doi.org/10.1002/0470845899

- Crecraft DI, Gergely S (2002) Analog Electronics: Circuits, Systems and Signal Processing ( $1^{\text {st }}$ edn). Butterworth-Heinemann, $425 \mathrm{pp}$ https://doi.org/10.1016/B978-075065095-3/50001-5

- Gerstner W, Kistler WM (2002) Spiking Neuron Models: Single Neurons, Populations, Plasticity. Cambridge University Press, 496 pp. https://doi.org/10.1017/CBO9780511815706
Gottlieb I (1998) Practical Transformer Handbook. Elsevier Science \& Technology, $192 \mathrm{pp}$.

- Gray PR, Hurst PJ, Lewis SH, Meyer RG (2009) Analysis and Design of Analog Integrated Circuits ( $5^{\text {th }}$ edn). JohnWiley \& Sons, Inc., 896 pp.

- Greshilov AA (2014) Mathematical Methods of Decision-Making ( $2^{\text {nd }}$ edn). MGTU Publ., Moscow, 647 pp.

- Gupta RB (2008) Hydrogen Fuel: Production, Transport, and Storage. CRC Press, 624 pp. https://doi.org/10.1201/9781420045772

- Haykin S (1999) Neural Networks - A Comprehensive Foundation. $\left(2^{\text {nd }}\right.$ edn). Prentice Hall Inc., 1104 pp. 
- Maass W, Bishop CM (2001) Pulsed Neural Networks. A Bradford Book, 377 pp.

- Moss F, Gielen S (2001) Neuro-informatics and Neural Modelling. North Holland, 1080 pp. https://doi.org/10.1016/S13838121(01)80002-6

- OECD (2006) Nuclear Production of Hydrogen: Nuclear Science. Third Information Exchange Meeting, Oarai, Japan 5-7 October 2005, Organisation for Economic Co-operation and Development, Nuclear Energy Agency. OECD Publishing, 414 pp. https://www. oecd-nea.org/science/pubs/2004/5308-production-hydrogen.pdf

- Saleh Bahaa EA, Teich MC (2007) Fundamentals of Photonics. Wiley-Interscience, $1200 \mathrm{pp}$.
- Sterratt D, Graham B, Gillies A, Willshaw D (2011) Principles of Computational Modelling in Neuroscience. Cambridge University Press, 404 pp.

- Strongin RG, Gergel VP, Grishagin VA, Barkalov KA (2013) Parallel Computations in Global Optimization Problems. MGU Publ., Moscow, 280 pp. [in Russian]

- Tomilova AP (1984) Applied Electrochemistry. Textbook for high schools. Khimiya Publ., Moscow, 520 pp.

- Wai-Kai C(2005) Nonlinear and Distributed Circuits. CRC Press, 352 pp.

- Yan XL, Hino R(2011) Nuclear Hydrogen Production Handbook. Series: Green Chemistry and Chemical Engineering. CRC Press, 939 pp. https://doi.org/10.1201/b10789-4 